\title{
Multi-tissue methylation clocks for age estimation in the common
}

\section{bottlenose dolphin}

Todd R. Robeck ${ }^{1 \#}$, Zhe Fei ${ }^{2}$, Amin Haghani ${ }^{3}$, Joseph A. Zoller ${ }^{2}$, Caesar Z. Li $^{2}$, Karen J. Steinman ${ }^{5}$, Stacy DiRocco ${ }^{6}$, Lydia Staggs ${ }^{6}$, Todd Schmitt ${ }^{7}$, Steve Osborn ${ }^{8}$, Gisele Montano ${ }^{5,6}$, Magdalena Rodriguez ${ }^{9}$, Steve Horvath ${ }^{2,3 \#}$

$\underline{\text { Affiliations }}$

${ }^{1}$ Zoological Operations, SeaWorld Parks and Entertainment, 7007 SeaWorld Drive, Orlando, Florida, USA;

${ }^{2}$ Department of Biostatistics, Fielding School of Public Health, University of California, Los Angeles, Los Angeles, California, USA;

${ }^{3}$ Department of Human Genetics, David Geffen School of Medicine, University of California, Los Angeles, Los Angeles, California, USA;

${ }^{5}$ Species Preservation Laboratory, SeaWorld San Diego, 2595 Ingraham Rd., San Diego, California, USA;

${ }^{6}$ SeaWorld Orlando, 7007 SeaWorld Drive, Orlando, Florida, USA;

${ }^{7}$ SeaWorld San Diego, 500 SeaWorld Drive, San Diego, California, USA;

${ }^{8}$ SeaWorld San Antonio, 10500 SeaWorld Drive, San Antonio, Texas, USA;

${ }^{9}$ Miami Seaquarium, 4400 Rickenbacker Causeway, Miami, Florida, USA

\#Corresponding authors

Steve Horvath, PhD, ScD E-mail: shorvath@mednet.ucla.edu

Todd Robeck, PhD, DVM E-mail: todd.robeck@seaworld.com

Key words: Tursiops truncatus, bottlenose dolphin, epigenetic clock, DNA methylation 


\begin{abstract}
Accurate identification of individual ages within wild bottlenose dolphins (Tursiops truncatus) is critical for determining population health and the development of population management strategies. As such, we analyzed DNA methylation patterns by applying a custom methylation array (HorvathMammalMethyl40) to both blood $(\mathrm{n}=140)$ and skin samples $(\mathrm{n}=87)$ from known age or approximate age (0 to 57 years) bottlenose dolphins. We present three bottlenose dolphin specific age estimation clocks using combined blood and skin (48 CpGs, $\mathrm{R}=$ 0.93, median absolute error $=2.13$ years $)$, blood only (64 CpGs, $\mathrm{R}=0.97$, error $=1.46$ years $)$ and skin only (39 CpGs, $\mathrm{R}=0.95$, error= 2.53). Our sex estimator based on $71 \mathrm{CpGs}$ predicts the sex of any odontocete species with $99.5 \%$ accuracy. We characterize individual cytosines that correlate with sex and age in dolphins.

The presented epigenetic clocks are expected to be useful for conservation efforts and for studying anthropogenic events.
\end{abstract}




\section{INTRODUCTION}

Accurate age estimation of wild cetaceans is an important component of any population health assessment and is critical for the development of management plans designed to help wild populations in need[1]. For bottlenose dolphins, standard age estimations of wild animals rely on a combination of techniques including animal length (allometry), long term "capture and recapture" of animals using photo identification, and the counting of tooth growth layer groups (GLGs). Age estimators based on allometry lose accuracy once physical maturity has been reached and surpassed[2,3]. Although long term photo identification studies have produced the most robust and accurate data concerning life history of a few populations of bottlenose dolphins, these studies face limitations, e.g. the inability to accurately define animals that had already reached physical maturity at the start of the surveys[4, 5]. Although long-term photo identification programs have been used with a few populations of bottlenose dolphins, their high economic costs and long-term time commitments render them less effective for rapid and time-sensitive evaluations of critically endangered or threatened populations.

Ages of odontocetes can be estimated based on tooth growth layer groups (GLGs[6]). Although GLGs represent the current gold standard for determining the age of bottlenose dolphins, the requirement for a tooth may render this method unacceptably invasive for many research efforts. Finally, some debate exists concerning the accuracy of relying on growth ring deposition for age estimation older animals that may have experienced significant tooth wear[7].

As such, other aging methods have been evaluated for use with bottlenose dolphins or other cetaceans including eye lens aspartic acid racemization[8, 9], fatty acid composition[10-12], radiocarbon 14 dating from fallout[13], telomere length[14], radiograph changes in tooth to pulp ratio[15] and pectoral flipper bone ossification[16]. However, all methods vary in inherent 
accuracy and must be calibrated against some age estimate of the species in question, most often by using GLG counts, and each have limitations for field use and accuracy across different age classes.

Recent efforts at developing less invasive methods with enhanced accuracy for age determination across multiple species, including marine mammals, has led to a surge in the application of species-specific DNA methylation profiles for the development of epigenetic aging clocks[1, 3, 17-23]. DNA methylation (DNAm) is described as an epigenetic modification whereby a transfer of a methyl $\left(\mathrm{CH}_{3}\right)$ group from $\mathrm{S}$-adenosyl methionine (SAM) to the fifth position of cytosine nucleotides, forming 5-methylcytosine $(5 \mathrm{mC})$ nucleotides[24]. The degree of methylation, hypo or hyper can be highly correlated with both chronological age and in the case of abnormal physiological conditions, accelerated aging[25-28].

An epigenetic aging clock was recently published for the bottlenose dolphin (BEAT). The BEAT clock, which relied on methods described for the prior development of a humpback whale specific aging clock, screened $17 \mathrm{CpG}$ sites located within DNA that were harvested from biopsied bottlenose dolphin skin samples to identify two CpGs associated with two genes (TET2: CpG site 2, and GRIA2: CpG site 5) that have a high correlation $\left(\mathrm{R}^{2}=0.779\right)$ with chronological age, error within \pm 4.8 years $[3,17]$. In addition to the bottlenose dolphin specific BEAT clock, we recently published a multi-tissue Odontocete Epigenetic Aging Clock (OEAC) which accurately (estimates age from blood or skin samples within any odontocete species[1]. The OEAC was developed by applying the same custom DNA Infinium methylation array (HorvathMammalMethylChip40) to skin and blood samples from nine different known age odontocete species, including bottlenose dolphins[1]. Although this odontocete clock works impressively well (median $\mathrm{R}=0.95$, median absolute error estimation of 2.6 years), evidence suggests that both correlation and accuracy could 
be improved within each individual species by developing species-specific clocks with an increased sample size for the tissues of interest [29, 30]. For wild bottlenose dolphins, the majority of tissues sampled are collected via remote biopsy and include both skin and blubber samples. Blubber cells are often used to determine hormone concentrations and to evaluate the bioaccumulation of organochloride toxins, while DNA from skin samples is often used to determine relatedness among conspecifics[31-33]. Thus, there is an existing, relatively large historical collection of skin samples and precedent for future collection of skin samples that could also be used for age determination.

In addition to clocks for chronological age determination, recent work in humans has indicated that DNAm clocks can be useful for detecting epigenetic accelerated aging (defined as discrepancy between epigenetic and chronologic age), and that the detection of an accelerated age condition can be predictive of multiple disease states and mortality[34-37]. Epigenetic clocks for bottlenose dolphins may be promising tools for quantifying adverse effects that anthropogenic environmental stressors are creating in wild populations [21].

Therefore, the objectives of our research were 1) to apply the mammalian methylation array technology to develop highly accurate bottlenose dolphin clocks based on blood and/or skin samples; 2) top characterize significant aging associated $\mathrm{CpGs}$ identified via epigenome-wide association studies (EWAS); and 3) to characterize sex linked CpGs, and 4) to develop sex estimators for odontocetes based on DNA methylation data.

\section{Results}


We obtained DNA methylation profiles from blood $(\mathrm{n}=140)$ and skin $(\mathrm{n}=87)$ samples from 140 animals (44 males and 96 females) ranging in ages from zero to 57 years of age (mean: 19.8 y, median: 16.8 y). For each animal, we had either blood or skin samples or both. An unsupervised hierarchical analysis clustered the methylation arrays by tissue type (Supplementary Fig. 1).

\section{Epigenetic aging models}

We developed three bottlenose dolphin clocks for i) blood + skin, ii) blood only, and iii) skin only (Fig. 1). To arrive at unbiased estimates of the accuracy, we used Leave One Out Cross Validation (LOOCV). We assessed the correlation between predicted age and chronological age, $\mathrm{R}$, and the median absolute error (MAE). LOOCV reveals that all 3 dolphin clocks are highly accurate: blood and skin clock: $\mathrm{R}=0.93, \mathrm{MAE}=2.13$ years, blood clock: $\mathrm{R}=0.97 ; \mathrm{MAE}=1.46$ years, skin clock: $\mathrm{R}=0.95 ;$ MAE $=2.53$ years $($ Fig $1 . \mathrm{b}, \mathrm{d}, \mathrm{f})$.

The final versions of the three clocks (based on all training data) involve 48 CpGs (skin and blood clock), 64 CpGs (blood clock), and 39 CpGs (skin clock) as detailed in Supplementary Table 1. The 3 clocks don't share any CpGs in common (Supplementary Fig. 2). Similarly, the blood clock and the skin clock only share $1 \mathrm{CpG}$ in common (Supplementary Fig. 2).

\section{Methylation estimator of sex}

Methylation based estimators of sex can be useful for identifying human errors in sample labelling or DNA processing. To define a methylation based sex estimator for dolphins and other odontocetes we combined our data with those from 8 additional odontocete species[1]. The sex estimator was developed by fitting a binomial elastic net regression model on $n=447$ odontocetes samples $(\mathrm{n}=276$ female and $\mathrm{n}=171$ male samples). The model only misclassified two male killer 
whale samples (99.5\% accuracy, Table 2$)$. The 71 CpGs of the sex estimator most likely map to sex chromosomes in odontocetes because they map to human sex chromosomes (based on human genome hg19).

\section{Epigenome-Wide Association Studies of age}

In total, only 23,005 probes from the mammalian array (HorvathMammalMethylChip40) could be mapped to the bottlenose dolphin genome assembly (turTru1.100). These probes are adjacent to 4558 unique genes: $65 \%$ in gene bodies, $10 \%$ in promoters, and $25 \%$ in distal regions. The number of aligned probes in the bottlenose dolphin is much lower than other cetacean species (e.g., 34,358 probes in the killer whale[1]), which probably reflects the lower quality of the dolphin genome assembly. A total of 6073 and 604 CpGs were significantly correlated with age in blood and skin, respectively $\left(\mathrm{p}<10^{-4}\right.$, Fig. $2 \mathrm{a}$ ). This difference in $\mathrm{CpG}$ counts between sample types reflects differences in statistical power and sample size (blood, $\mathrm{n}=140$, age range: 1-57 y; skin, $\mathrm{n}$ $=87$, age range: $4-57$ years). The top age-related changes in blood included hypermethylation in LHFPL4 exon and HOXC4 promoter. In skin, the CpGs with the strongest positive correlation with age were located downstream of EVX2 and in an exon of NOL4 exon (Table 1). CpGs in promoters largely gained methylation with age, which matches DNAm aging pattern in other mammals (Fig. 2b). CpGs located in CpGs islands had higher age correlations than CpGs located outside of islands (Fig. 2c).

We found that 94 CpGs (64 hypermethylated, 30 hypomethylated) have a significant correlation with age in both blood and skin (Fig. 2d). Aging effects in blood were moderately correlated with those in skin $(\mathrm{R}=0.27$, Fig. $2 \mathrm{e})$. Functional enrichment studies reveal that significant age-related $\mathrm{CpGs}$ are located near genes that play a role in development and with 
polycomb repressor complex 2 target genes H3K27ME3, EED, PCR2, and SUZ12 (Supplementary

Fig. 3, Table 1). Many CpGs correlate with age in a tissue specific manner. As an example, while HOXC4 promoter, suspected to be involved with mylo-proliferative disorders and skin tumors[38, 39], is hypermethylated with age in blood $(\mathrm{z}=4.7)$, it was hypomethylated in skin $(\mathrm{z}=-4.5)$.

\section{Sex specific DNAm patterns in bottlenose dolphins}

Our dataset allowed us to characterize sex differences in baseline methylation levels, and, also, sex effects on aging rates. At a nominal/unadjusted significance threshold of $\mathrm{p}<10^{-4}, 1738$ and 742 CpGs were significantly associated with sex in dolphin blood and skin, respectively (Fig. 3a). Due to the incomplete genome assembly we were not able to determine whether these CpGs are located on sex chromosomes in bottlenose dolphins. However, we expect that these CpGs are located on sex chromosomes since more than $70 \%$ of these CpGs in blood and $90 \%$ in skin were located on the human X chromosome. Some of the top sex-related gene regions are as follows: blood, PTCHD1 exon (hypermethylated in females) and HUWE1 exon (hypomethylated in females); skin, HUWE1 exon (hypomethylated in females) and CNKSR2 exon (hypermethylated in females).

Aging effects were highly correlated between the sexes both in blood $(\mathrm{R}=0.78)$ and skin $(\mathrm{R}$ = 0.62, Fig. 3B). We highlight a few CpGs with distinct sex specific aging patterns in Fig. 4.

\section{Discussion}

This study describes the development of a three highly accurate bottlenose dolphin DNAm epigenetic aging clocks using combined blood and skin, blood only or skin only that were 
developed with samples collected from known (88\%) or approximately known age animals. The mammalian methylation array used in this research profiles cytosines that are conserved across mammalian species $[1,22]$.

Similar to our recently published odontocete epigenetic aging clock (OEAC[1]), the data were mainly comprised of animals whose ages were known precisely (often the birthday was known) or whose age could be estimated with high accuracy. As such, this dolphin clock is expected to become a useful molecular method for accurate age measurement in dolphins.

Compared to recent efforts to develop rapid, non-invasive methods for age determination, including, pectoral flipper bone ossification and dental radiographic determination of the pulp to tooth area ratio which appear to be accurate in juvenile animals $[15,16]$. Although bone ossification and pulp to tooth ratios benefit from portability of radiography units that can be used in the field to estimate age within hours of image collection, they still require animal restraint and suffer from reduced accuracy with animals that have reached physical maturity[15, 16]. By contrast, epigenetic clocks apply to the entire life-span of the bottlenose dolphin [1].

Two previous epigenetic clocks apply to Bottlenose dolphins: Bottlenose dolphin Epigenetic Aging estimation Tool (BEAT) and the Odontocete Epigenetic Aging Clock (OEAC)[1, 3]. The OEAC clock is the first multi-tissue clock for multiple odontocetes species. For this clock, nine different odontocete species were used across 446 blood and skin samples, and within this data set, there were 181 bottlenose dolphin samples (140 blood and 41 skin samples). Although we obtained very accurate predictive results for the bottlenose dolphin blood ( $\mathrm{R}=0.95$, MAE $1.5 \mathrm{yr})$ and skin samples $(\mathrm{R}=0.91$, MAE $4.8 \mathrm{yr})$ in our OEAC, predictions based on skin samples were below accuracy levels with a wider variance (MAE) than was found in blood. While still within ranges currently used in forensic sciences for age estimation to within a decade [40-42], this 
increase in variance in skin samples as compared to blood was hypothesized to be primarily due to a reduced sample size as compared to blood (40 vs 140). Additionally, other variables that may have contributed toward increased predictive variance may have included the types and proportion of cells collected during each skin collections (for discussion see Robeck et al.[1]) and may possibly reflect a higher sensitivity for skin cells to experience varying epigenetic methylation rates across age [43]. Therefore, we hypothesized that by increasing our skin sample size for bottlenose dolphins, we could improve our predictive ability within skin samples over our previous results presented with the OEAC development[1]. Thus, in our present study, we increased our skin sample size by over $100 \%$ and obtained an almost a 50\% reduction in MAE (2.5 vs 4.8) and brought our median predictive ability from skin samples across all age groups to within 5 years $( \pm$ $2.5 \mathrm{y})$. Although these results cannot take away from the accuracy and application of the OEAC across multiple species, they do demonstrate the superior accuracy of species-specific clocks.

Direct comparisons of the accuracy between the BDAC and the OEAC are possible because approximately half of the samples were independently analyzed by each clock. However, direct comparison against the only other bottlenose dolphin clock, the BEAT is not possible due to different samples and measurement platforms. In general, our BDAC skin clock had improved accuracy $(\mathrm{R}=0.95, \mathrm{MAE}=2.5$ years $)$ at predicting ages of bottlenose dolphins when compared to the BEAT results (BEAT, $\mathrm{R}^{2}=0.74$, root mean square error $=5.1$ years[3]). Important differences between the BDAC and the BEAT clock that have been identified as limitations toward methylation clock development include: (1) the sample number used for the BEAT clock was $\sim$ half $(n=40)$ the number used for the BDAC; and 2) and the number of CpGs used for final clock development were vastly reduced (2 versus 39$)$. As we have already demonstrated through direct comparisons between the BDAC and OEAC and as was hypothesized by the developers of the 
BEAT clock, increased sample size has direct effects on clock accuracy[3]. In addition to sample size, by filtering through a dramatically larger catalog of CpGs we were able to identify and incorporate a wider array of significant age related $\mathrm{CpG}$ sites into the predictive model resulting an improved representation of organism age $[22,29,30]$.

The BEAT clock [3] identified two CpG sites on two genes, TET2 (CpG site 2) and GRIA2 (CpG site 5) that accounted for $78 \%$ of the age-related variation in $\%$ DNA methylation. These same genes were also found to correlate with age in humpback whales[17]. Although we had several probes that mapped to the genomic regions of GRIA2, this gene was not considered to be within the top 10 genes that accounted for age-related methylation changes in neither our present study nor within the OEAC[1]. Our measurement platform (the mammalian array) did not cover the TET2 gene, and we only had probes that mapped to the genomic regions of GRIA2 gene. Interestingly, most of these GRIA2 probes (both exon and intron) were hypomethylated with age in both the blood and skin of bottlenose dolphins (Supplementary Fig. 4). This suggests that although promoter of GRIA2 gains methylation with age[3], selected CpGs in the gene body lose methylation with age.

Besides epigenetic clock development, the mammalian array is a uniquely reproducible tool for a direct genome-wide comparison of DNAm changes across cetaceans. Our EWAS identified DNAm aging CpGs proximate to genes associated with development (Supplementary Fig. 3). The top proximate hypermethylated $\mathrm{CpG}$ in blood was associated with $L H F P L 4$, a gene known to be involved with GABA regulation in the central nervous system and peripheral lipoma formation[44, 45]. This gene has recently been identified as the most predictive age-related gene across mammalian species and despite its conserved status, its contribution towards age-related functional decline has not be clarified[37]. The top two hypomethylated CpGs (both within introns) in the 
blood were proximal to $U B P 1$, a gene that is closely related to TFCP2 and is considered part of the Grainyhead family of transcription factors[46]. Although UBPI has been identified as involved in angiogenesis during growth and development and Alzheimer's disease, its redundancy and homology with TFCP2 make its yet unidentified role in cancer regulation a likely possibility[46, 47]. For skin, the top proximate genes were associated with development and energy metabolism (Supplementary Fig. 3). In addition to these $\mathrm{CpG}$ related genes, multiple CpGs, in both blood and skin, that were associated with genes within the PRC2 complex were hypermethylated (Supplementary Fig. 3). The PRC2 is required for embryonic stem cell differentiation but also plays a role in in aging $[48,49]$. Overall, we find that age-related methylation changes in bottlenose dolphins mirror those in other odontocete species and mammals in general $[1,37]$.

Our sex related CpGs were largely the same as those found in multiple odontocete species [1]. Therefore, we were able to use these CpGs to develop a highly accurate (99.5\%) multivariate predictor of sex across all odontocete species. Although not of primary importance for this research, the ability to determine sex in species that do not have easily identifiable sexual dimorphic patterns is an attractive property of cytosine methylation data.

Similar to previous work with other cetaceans, we found no evidence that accounting for sex was necessary for the development of the three BDAC clocks[1, 23]. Aging effects in males were highly correlated with those in females both in blood $(\mathrm{R}=0.78)$ and skin $(\mathrm{R}=0.62)$. While the majority of sex specific CpGs were found on the X chromosome (78.8\%, Supplementary Data File 1), the proximate genes associated with the top non $\mathrm{X}$ chromosome CpGs, which were different between the two sexes included in blood, related to cancer (PSMD7[50], BCOR[50]) and the central nervous system (FEZF2 [51]). Noteworthy sex related autosomal genes implicated by the skin methylation data play a role in DNA repair (POLA1[52]), neurodevelopment (TCF4[53]), oral 
cancer (SOX2[54], higher methylation rates in females) and nutritional or chemical mediated ER autophagy (CALCOCO1[55]). These results suggest a potential difference in aging phenotypes in dolphin sexes, however, this hypothesis should be examined in future studies.

In conclusion, we characterized cytosines that correlate strongly with age and sex in bottlenose dolphins. We present three highly accurate DNA methylation-based estimators of chronological age for bottlenose dolphins. The high accuracy of these clocks could not have been achieved without knowing the precise ages of a large number of animals which in turn relied on long term efforts of monitoring and caring for these animals.

\section{Materials and Methods}

\section{Ethics approval}

The study was authorized by the SeaWorld Parks and Entertainment and Miami Seaquarium animal care and use committee.

\section{Study Animals}

For model development, our study population included skin $(\mathrm{n}=50)$ and blood samples $(\mathrm{n}=$ 140) from 140 bottlenose dolphins located at three SeaWorld Parks (Orlando, San Antonio, and San Diego) and Discovery Cove (Orlando, FL). The known age animals consisted of 123 zoo born animals (38 male, 85 females) with a median age of $14.5 \mathrm{y}$ (range: 0.57 to $40.7 \mathrm{y}$ ), and 17 wild born animal (6 male, 11 females) with a median age of 36.4 years range: 3.9 to $57.0 \mathrm{y}$ ). Known 
$(87.9 \%)$ or estimated (based on length at capture or rescue for stranded animals) birth dates were used for correlating against methylation predicted age

\section{Sample collection}

Blood samples were collected either voluntarily from the peripheral periarterial venous rete on the ventral tail fluke using an 18 to 22 gauge winged blood collection set or attached to a vacutainer collection system during routine physical examination. Blood was collected by either the veterinary technician or staff veterinarian into BD Vacutainers (Becton Dickinson, Franklin Lakes, NJ) containing EDTA. Samples were inverted in the Vacutainer a minimum of 10 times and then frozen at $-80^{\circ} \mathrm{C}$ until further testing.

Skin scrapings were collected either under stimulus control or manual restraint using a sterile disposable dermal curette or 8.0 mm biopsy punch (Miltex, Integra Life Sciences Corp., York, PA) from a location just posterolateral of the dorsal fin overlying the epaxial muscle. Prior to collection, a cold pack was placed on the site for several minutes prior to sampling to numb the sample site. Skin samples were placed into sterile cryovials (Nunc® Cryotubes, Millipore Sigma Corp., St. Louis, MO) and stored at $-80^{\circ} \mathrm{C}$ until shipment on dry ice. Skin samples from non-living animals were obtained from frozen $\left(-80^{\circ} \mathrm{C}\right)$ specimens that had been previously collected and stored during standard necropsy procedures.

\section{DNA extraction}

Genomic DNA was extracted from clotted whole blood samples using QIAamp DNA Mini blood kit and following the manufacturer's instructions (Qiagen, Valencia, CA). Tissue samples were pulverized and broken down manually using a drill and DNA was extracted using DNeasy 
Tissue kit (Qiagen) and following the manufacturer's instructions with the exception of extending the proteinase $\mathrm{k}$ digestion. DNA was then extracted using the automated nucleic acid extraction platform, Anaprep (Biochain, Newark, CA) that utilizes a magnetic bead extraction process and Tissue DNA Extraction kit (Anaprep).

\section{DNA methylation data}

The mammalian DNA methylation arrays were profiled using a custom Infinium methylation array (HorvathMammalMethylChip40) based on 37,491 CpG sites as previously described[56]. Out of these sites, 1951 were selected based on their utility for human biomarker studies; these CpGs, which were previously implemented in human Illumina Infinium arrays (EPIC, 450K), were selected due to their relevance for estimating age, blood cell counts or the proportion of neurons in brain tissue. The remaining 35,540 probes were chosen to assess cytosine DNA methylation levels in mammalian species[57]. The subset of species for each probe is provided in the chip manifest file at the NCBI Gene Expression Omnibus (GEO) platform (GPL28271). Raw data was normalization using the SeSaMe method which assigned a beta value (0 to 1 ) for each methylation estimate corresponding to each probe used for every sample[58]. Beta values were indicative of methylation rates, with 0 indicating no gene copies were methylated. Unsupervised hierarchical clustering analysis based on the interarray correlation was used to identify technical outliers which were then removed from further analysis.

\section{Penalized regression}

Details on the clocks (CpGs, genome coordinates) and $\mathrm{R}$ software code are provided in the Supporting (SI) Appendix file. Penalized regression models were implemented with the R software 
package "glmnet"[59]. The optimal penalty parameters lambda in all cases were determined automatically by using a 10 fold internal cross-validation (cv.glmnet) on the training set. Alpha $=1 / 2$ corresponds to the elastic net penalty that penalizes the coefficients based on their magnitude. We performed a Leave One Sample Out Cross Validation (LOOCV) scheme for arriving at unbiased estimates of the prediction accuracy of the different DNAm aging clocks. The LOOCV, which is based on previously reported methods[60, 61], does the following for each of the $N$ samples: omit the one sample from the training set; fit the clock on the training set with $(N-1)$ samples; predict the DNAm age of the omitted sample with the fitted clock. Therefore, the LOOCV allows one to estimate the accuracy of predicting the age for any unknown bottlenose dolphin sample by the clock. The cross-validation study reports unbiased estimates of the age correlation $\mathrm{r}$ (defined as Pearson correlation between the DNAm age estimate and chronological age) as well as the median absolute error (MAE). The accuracy of the resulting bottlenose dolphin clocks was assessed via LOOCV estimates of: 1) the correlation $r$ between the predicted epigenetic age and the actual (chronological) age of the animal; and 2) the median absolute error (MAE) between DNAm age and chronological age.

\section{Multivariate sex predictor}

To predict sex (binary variable with values $1=$ female, $0=$ male), we used a binomial generalized linear model, regularized by elastic net[62]. The glmnet alpha parameter was set to 0.5 , i.e. we used elastic net regression. The lambda parameter of the glmnet function was chosen using 10fold cross validation. As we observed a reasonably balanced female-male sample sizes in the data set, we set a neutral prediction rule for the binomial generalized linear model. If the predicted probability for a sample being female was greater than 0.5 , the sample was predicted to be female. 
We used all odontocetes samples previously used for DNAm clock develeopment[1] and the additional samples from bottlenose dolphins, reported herein, in the training data so that the resulting sex estimator will be broadly applicable to odontocetes species.

\section{Data availability}

The data will be made publicly available as part of the data release from the Mammalian Methylation Consortium. Genome annotations of these CpGs can be found on Github https://github.com/shorvath/MammalianMethylationConsortium.

\section{Code availability}

The R software code used for clock development are provided in the Supplement.

\section{Acknowledgements}

We thank individuals from all of our zoological parks that contributed to toward sample collection and curating for this research. Specifically, from SeaWorld California (SWC) we thank Dr. Elsburgh Clarke, Dr. Kelsey Herrick, Rachelle Pastorkovich, Sarah McMillen, Melinda Tucker, Kim Regan, and Wendy Ramirez. From SeaWorld of Texas, we thank Dr. Jennifer Russel and Tara Klimek. From SeaWorld of Florida, we thank Dr. Dana Lindemann, Dr. Claire Erlacher-Reid, Jacob Vandenberg, Cynthia O’neil, Megan Fox, Siobhan Diaz, Cynthia Reyes, Stephanie Smith and Heidy Clifford. Finally, we thank Species Preservation Laboratory research technicians Amanda McDonnel and Jacqueline Posy. This is a SeaWorld Parks and Entertainment technical manuscript number 2021-7.

\section{FUNDING}


bioRxiv preprint doi: https://doi.org/10.1101/2021.05.03.442523; this version posted May 4, 2021. The copyright holder for this preprint (which

was not certified by peer review) is the author/funder, who has granted bioRxiv a license to display the preprint in perpetuity. It is made available under aCC-BY 4.0 International license.

This work was supported by the Paul G. Allen Frontiers Group (SH).

\section{Conflict of Interest Statement}

$\mathrm{SH}$ is a founder of the non-profit Epigenetic Clock Development Foundation which plans to license several of his patents from his employer UC Regents. The other authors declare no conflicts of interest. 
Table 1. Epigenome-wide associated studies (EWAS) identification of top three hypo [-] and hyper [+] methylated age associated CpGs, its proximate gene, the epigenetic clock which included CpG site its coefficients.

\begin{tabular}{|c|c|c|c|c|c|c|c|c|}
\hline Tissue & CpG Id & $\begin{array}{l}\text { Gene } \\
\text { (TCPG) }\end{array}$ & Possible relevance & $\begin{array}{l}\text { Methylation } \\
\text { pattern }\end{array}$ & Location & $\begin{array}{l}\text { EWAS } \\
\text { Z Stat }\end{array}$ & Clock & $\begin{array}{l}\text { Model } \\
\text { Coeff }\end{array}$ \\
\hline & $\operatorname{cg} 03537184$ & & $\begin{array}{l}\text { Protein Transcription factors [tumor } \\
\text { suppression], Reproduction, Alzheimer's[46, }\end{array}$ & - & & -16.1458 & Blood & $\begin{array}{l}-0.5336 \\
-1.2565\end{array}$ \\
\hline Blood & $\operatorname{cg} 17599620$ & UBP1 & 47] & & Intron & -15.2636 & & \\
\hline Blood & $\operatorname{cg} 15675612$ & HECW2 & $\begin{array}{l}\text { Neurodevelopment disorders, proteasomal } \\
\text { degradation }[63,64]\end{array}$ & - & Exon & -13.3931 & Blood & -0.0264 \\
\hline Blood & $\operatorname{cg} 22591675$ & NPAS3 & Neurodevelopment[65] & - & upstream & -13.220833 & Blood & NS \\
\hline Blood & $\operatorname{cg} 11084334$ & LHFPL4 & $\begin{array}{l}\text { Lipoma HMGIC fusion partner, GABA } \\
\text { regulation }[44,45]\end{array}$ & + & Exon & 18.6293 & Blood & 0.9011 \\
\hline Blood & $\operatorname{cg} 11716762$ & $\mathrm{HOXC} 4$ & Developmental, myeloid leukemia[38, 39] & + & Promoter & 16.9433 & Blood & 0.1355 \\
\hline Blood & $\operatorname{cg} 18304538$ & TBCA & $\begin{array}{l}\text { Protein folding cell structure, myopathy, } \\
\text { neuronal degeneration[66] } \\
\text { Chondrogenesis, collagen formation[67] }\end{array}$ & + & upstream & 15.3917 & $\begin{array}{l}\text { Blood, } \\
\text { BL+SK } \\
\text { Skin, }\end{array}$ & 1.0467 \\
\hline Skin & $\operatorname{cg} 13237109$ & SOX5 & & & Intron & -9.2044 & $\mathrm{BL}+\mathrm{SK}$ & \\
\hline Skin & $\operatorname{cg} 16947316$ & NUAK1 & Regulates cellular senescence, cancer $[68,69]$ & - & Exon & -9.0794 & Skin & -0.2563 \\
\hline Skin & cg20315976 & ETV1 & Prostate cancer $[70,71]$ & - & Promoter & -9.0276 & Skin & -0.9730 \\
\hline Skin & $\operatorname{cg} 09227056$ & EVX2 & Development, Gastric cancer[72, 73] & + & downstream & 10.2770 & Skin & 1.6972 \\
\hline Skin & $\operatorname{cg} 00200899$ & NOL4 & Tumor suppression gene $[74,75]$ & + & Exon & 9.3474 & Skin & 1.0239 \\
\hline Skin & cg20233387.1 & LHX8 & Neuronal development and oogenesis[76, 77] & + & upstream & 8.8417 & Skin & 0.8323 \\
\hline
\end{tabular}


Table 2. Elastic Net penalized linear model sex predictions based on odontocetes*, $n=447[1]$

\section{Female Samples}

Male Samples

\begin{tabular}{lrr}
\hline Predicted as Females & 276 & 2 \\
Predicted as Males & 0 & 169 \\
\hline True Positive Rates: & $100 \%$ & $98.83 \%$ \\
\hline
\end{tabular}

*Tissues from odontocete species used in the develop of this sex predictor included: bottlenose dolphin (Tursiops truncatus), beluga (Delphinapterus leucas), killer whale (Orcinus orca). Pacific white-sided dolphin (Lagenorhynchus obliquidens), short-finned pilot whales (Globicephala macrorhynchus), rough-toothed dolphin (Steno bredanensis), Commerson's dolphin (Cephalorhynchus commersonii), common dolphin (Delphinus delphis), and harbor porpoise (Phocoena phocoena). 

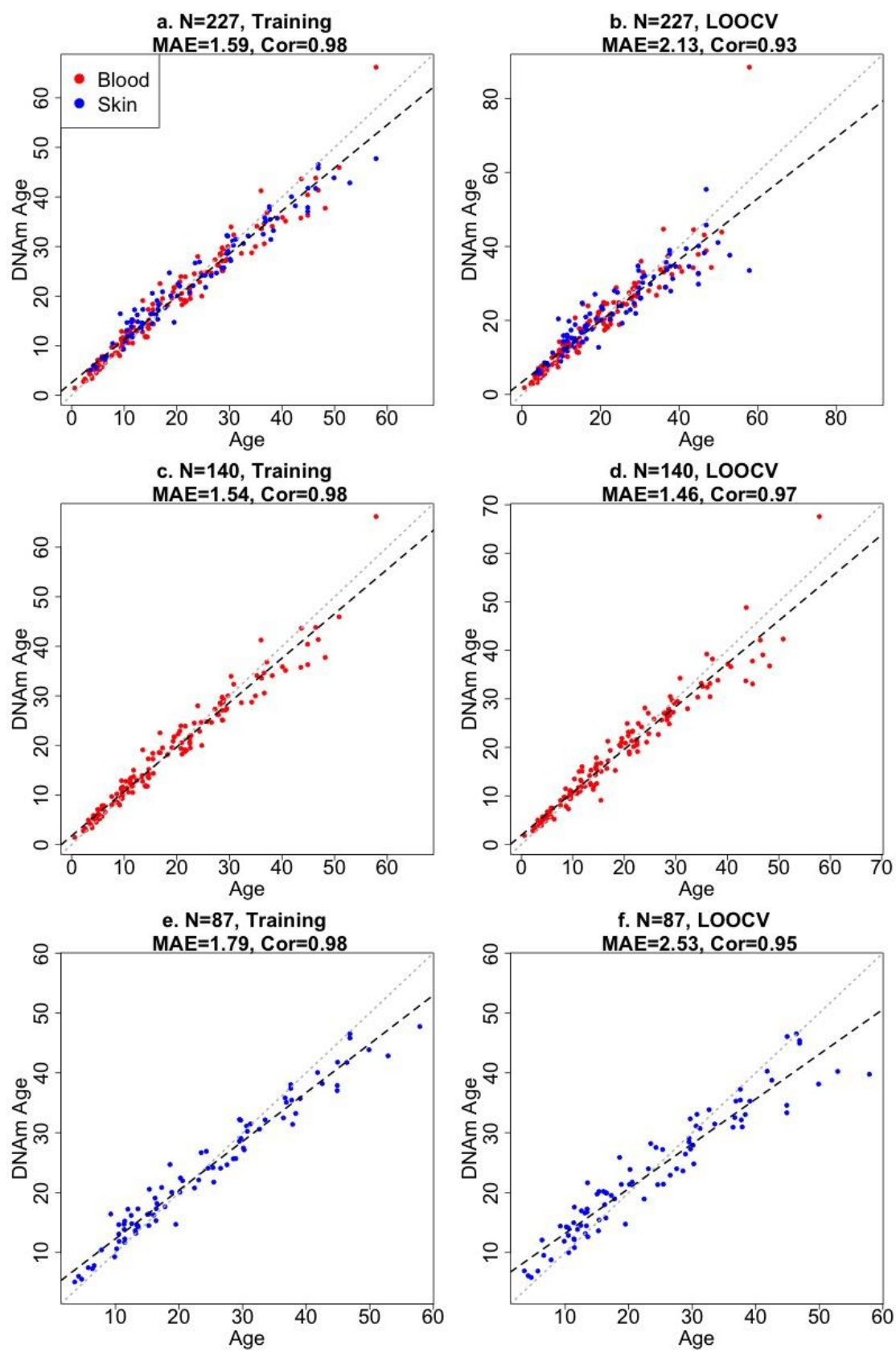

Fig 1. Accuracy of epigenetic clocks for bottlenose dolphin.

The panels correspond to three different clocks for bottlenose dolphins: multi tissue clock $(a, b)$; blood clock (c, d); skin clock (e, f) and the age estimates based on training (a, c, e) and LOOCV $(b, d, f)$. Dots are colored by tissue type ( $r e d=$ blood, blue $=$ skin $)$. The training set estimates (left panels) are highly biased. Each panel depicts a linear regression line (black dashed line), a diagonal line $(\mathrm{y}=\mathrm{x}$, dotted line), the sample size $(\mathrm{N})$, Pearson correlation (Cor) across all samples, median absolute error (MAE) across species. Chronological age (x-axis) and DNA methylation age estimates (y-axis) are in units of years. 


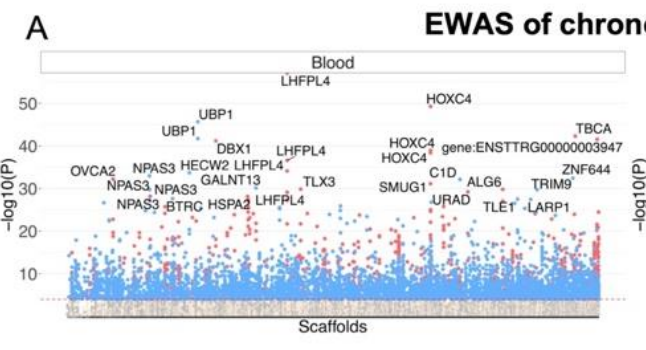

B Direction
of association

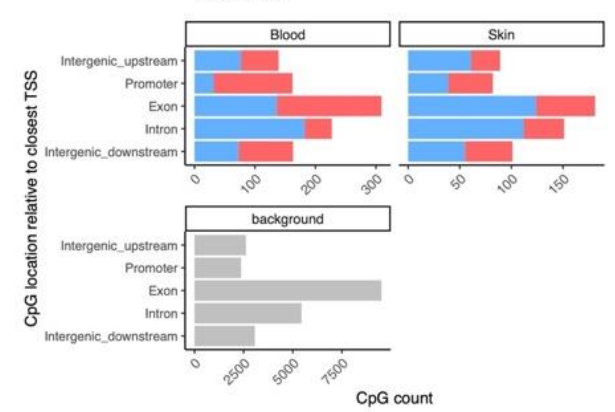

C
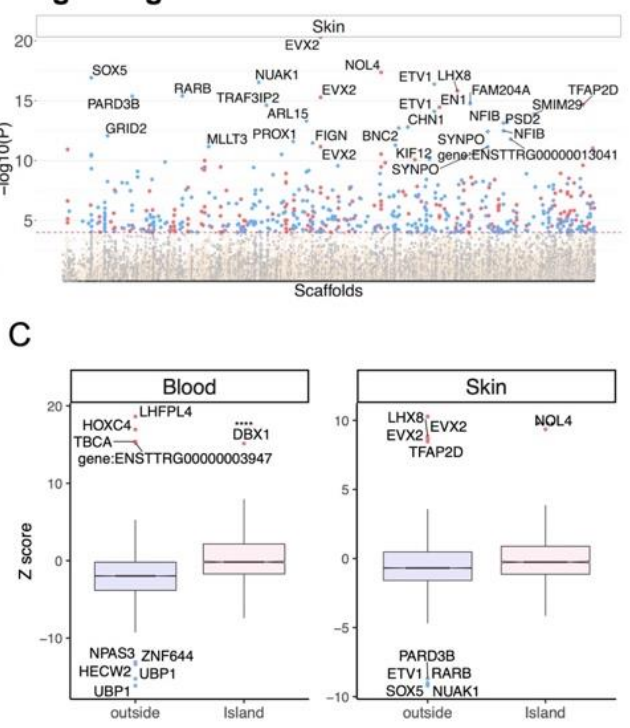

D

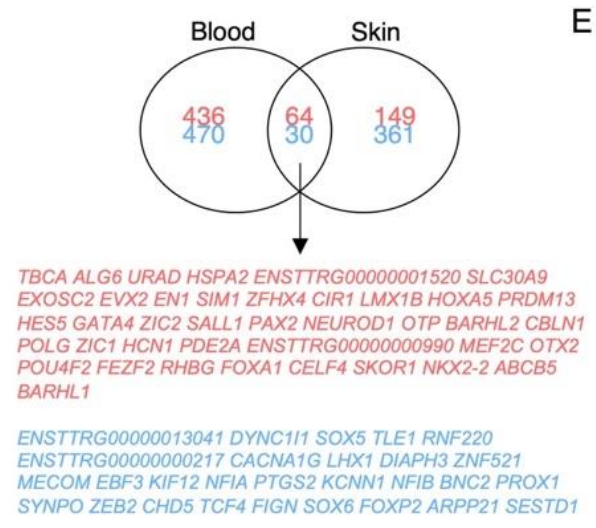

E

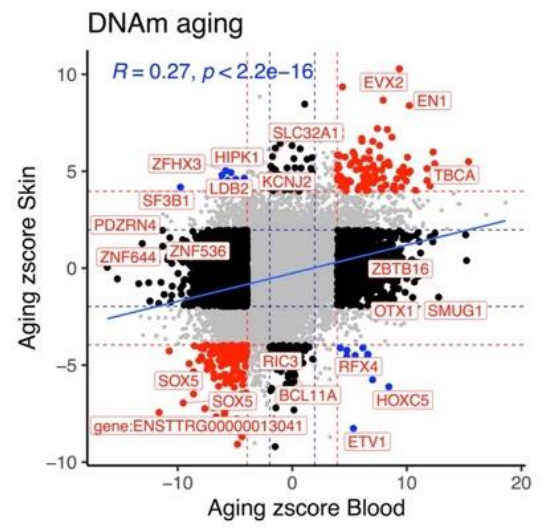

Fig 2. Epigenome-wide association (EWAS) of chronological age in skin and blood of bottlenose dolphin species

(A) Manhattan plots of the EWAS of chronological age. The coordinates are estimated based on the alignment of Mammalian array probes to the bottlenose dolphin (turTru1.100) genome assembly. The direction of associations with $\mathrm{P}<0.0001$ (red dotted line) is highlighted by red (hypermethylated) and blue (hypomethylated) colors. The 30 most significant CpGs were labeled by their respective neighboring genes. (B) Genomic location analysis of significant $\left(\mathrm{P}<10^{-4}\right)$ CpGs. The blood analysis focused on the top 500 positively age related CpGs and the top 500 negatively age related CpGs. The skin analysis focused on 213 positively and 391 negatively age related CpGs. (C) Box plot visualizing the median value (line inside the box) and the 25th and 75 th percent quartiles (top and bottom of the box), and the whiskers correspond to the $90 \%$ 
percentile. The $\mathrm{x}$-axis corresponds to $\mathrm{CpG}$ island status. (D) Venn diagram of the most significant age related CpGs in blood and skin. (E) Sector plot of DNA methylation aging effects in blood and skin. Each axis reports the $\mathrm{Z}$ statistic from a correlation test which follows a standard normal distribution under the null hypothesis. Red dotted line: $\mathrm{P}<10^{-4}$; blue dotted line: $\mathrm{p}>0.05$; Red dots: shared CpGs; black dots: tissue specific changes; blue dots: CpGs where age correlation differs between blood and skin tissue. The grey color in the last panel represents the location of 23,005 mammalian array probes mapped to the bottlenose dolphin (turTru1.100) genome

A
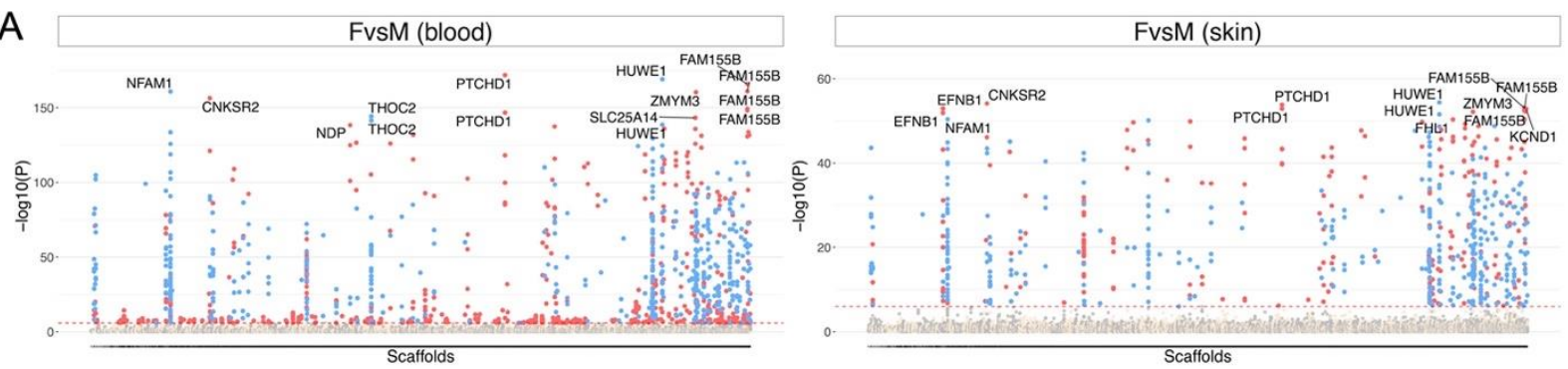

B
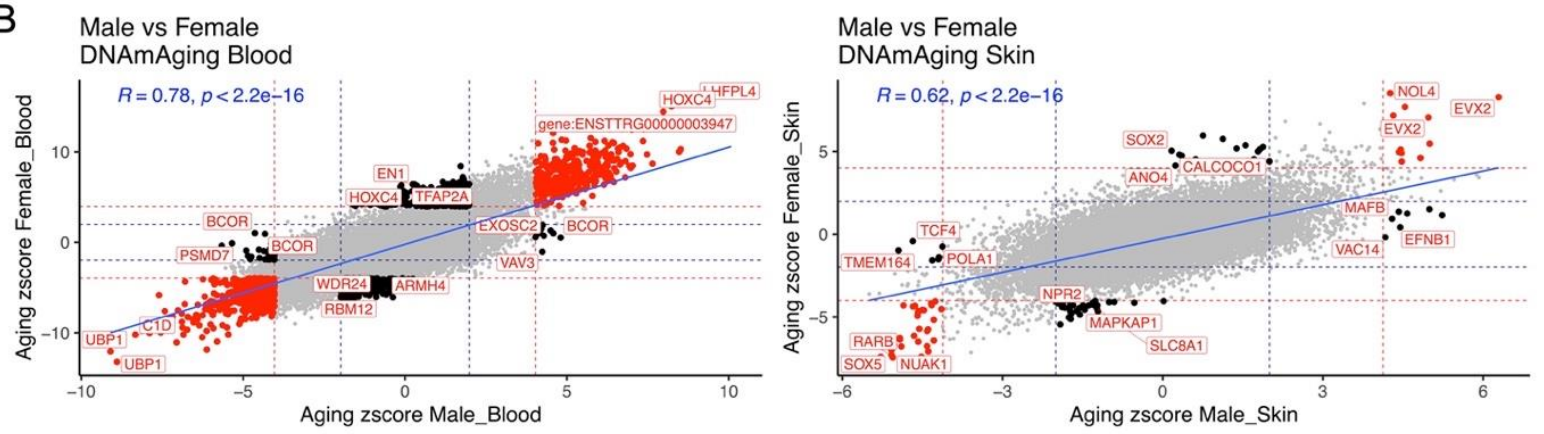

Fig. 3. Aging effects in male and female dolphins. A) Manhattan plot of the epigenome-wide association studies (EWAS) of sex. We adjusted the analysis for chronological age. Sample size: males' blood, 45; females blood, 95; males skin, 29; females skin, 58. The genome coordinates are estimated based on the alignment of Mammalian array probes to the bottle nose dolphin (turTru1.100) genome assembly. The horizontal red dotted line indicate a nominal/uncorrected significance level of $\mathrm{P}<10^{-4}$. Significant $\mathrm{CpGs}$ are colored in red if they are hypermethylated in females, blue hypomethylated in females. The 15 most significant $\mathrm{CpGs}$ are labeled by their respective neighboring genes. B) Sector plot of DNA methylation aging effects by sex in blood (left) and skin (right). Red dotted line: $\mathrm{P}<10^{-4}$; blue dotted line: $\mathrm{P}>0.05$; Red dots: shared CpGs; black dots: distinct changes between males and females.

61

62

63 

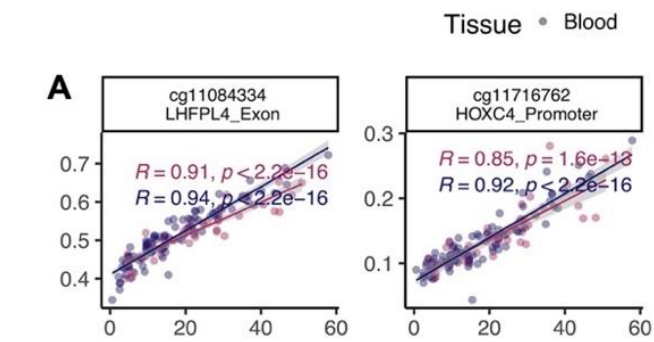

sex at Male Female
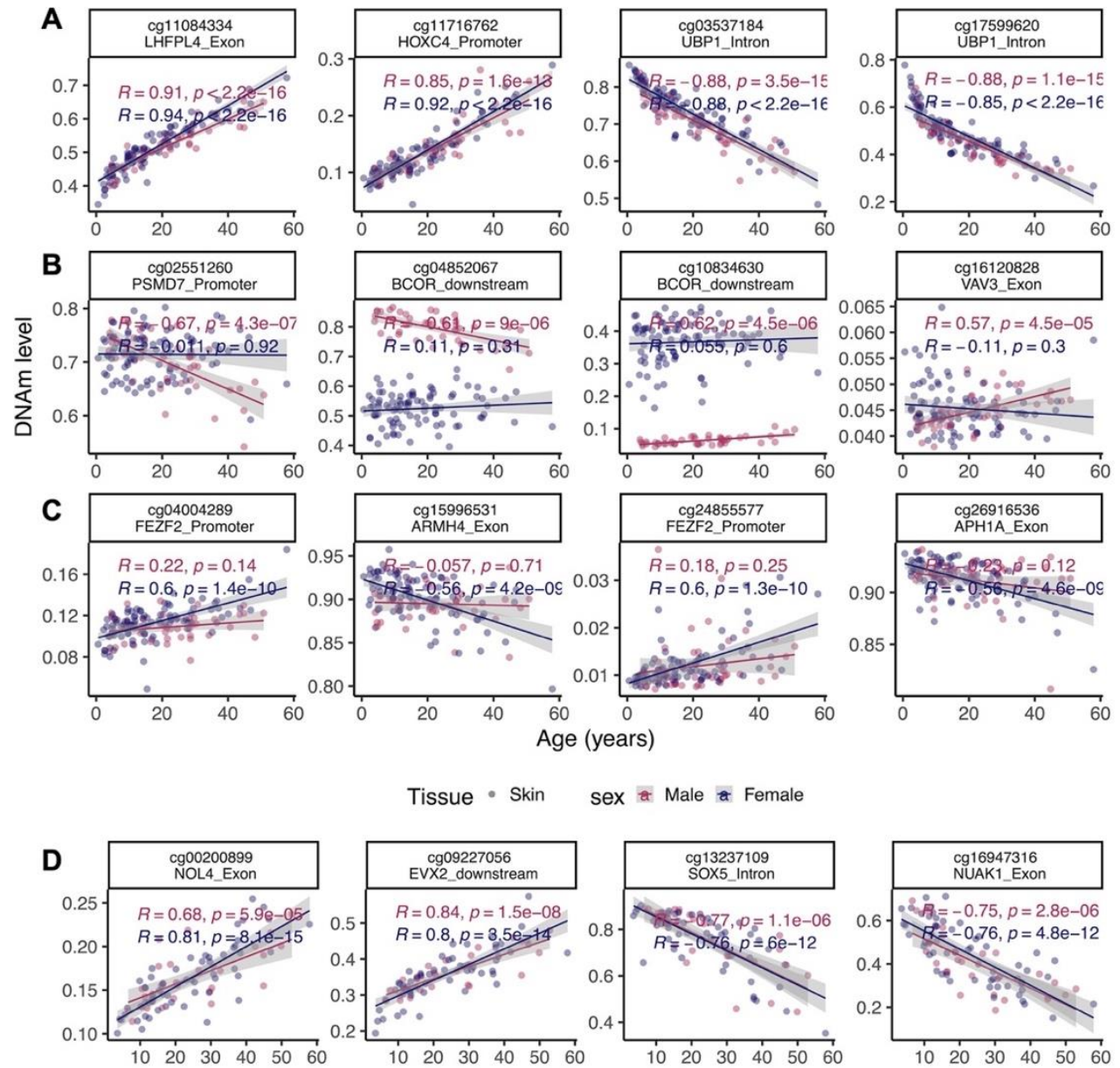

Tissue - Skin

sex Male Female
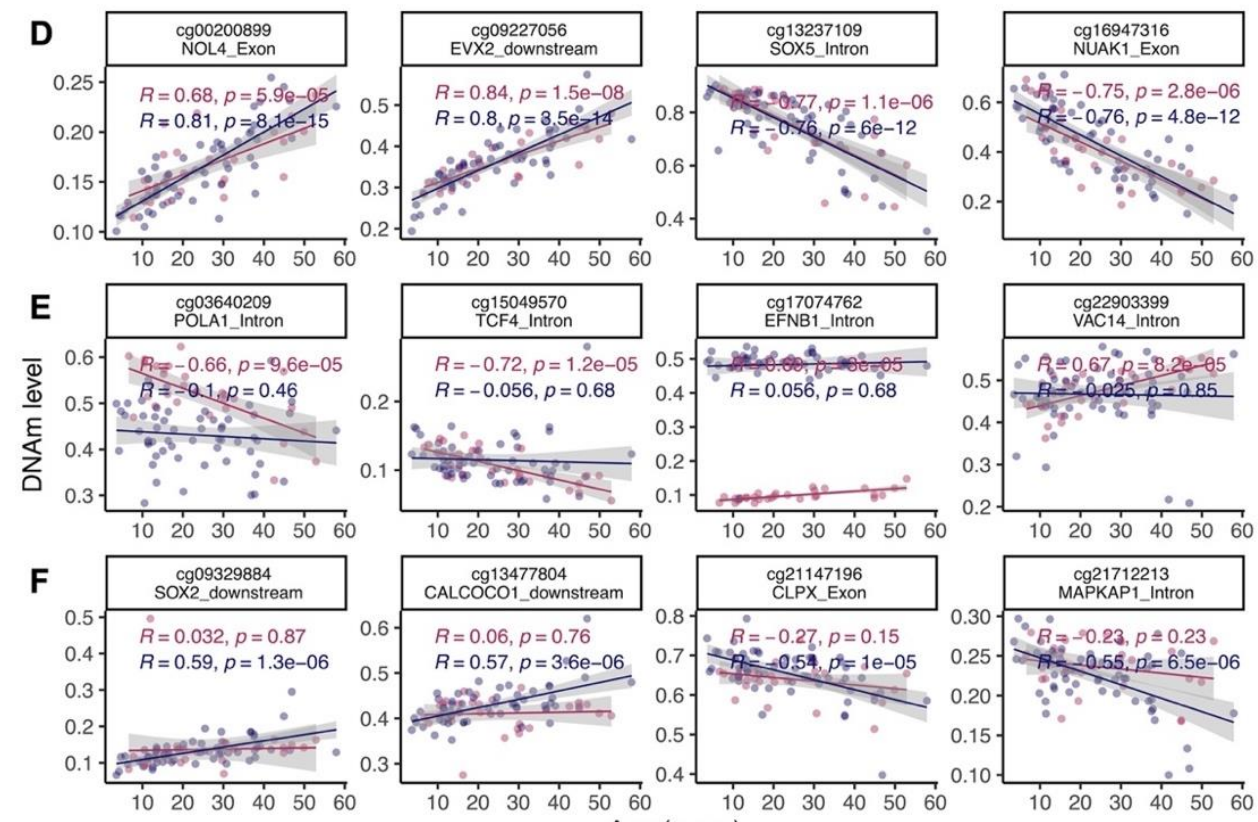

Age (years)

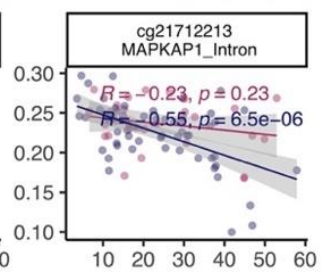

67 Fig. 4. Select age-related CpGs stratified by sex. (A) CpGs that change with age in male and

68 female blood samples from bottlenose dolphins. (B) Male specific changes in blood. (C) Female

69 specific changes in blood. (D) CpGs that change with age in both male and female skin. (E) Male

70 specific changes in skin. (F) Female specific changes in skin. The ordinary least squares

71 regression lines were carried out in males (red) and females (blue) separately. 


\section{References}

73 1. Robeck TR, Fei Z, Lu AT, Haghani A, Jourdain E, Zoller JA, Li CZ, Steinman KJ, DiRocco S,

74 Schmitt TL, Osborn S, Van Bonn B, Katsumata E, et al. Multi-species and multi-tissue methylation clocks for age estimation in toothed whales and dolphins. Communications

76 Biology. in press.

$772 . \quad$ McFee W. Age Distribution and Growth of Two Bottlenose Dolphin (Tursiops truncatus)

78 Populations from Capture-Release Studies in the Southeastern United States. Aquatic

79 Mammals. 2012; 38(1):17-30.

80 3. Beal AP, Kiszka JJ, Wells RS and Eirin-Lopez JM. The Bottlenose Dolphin Epigenetic Aging 81 Tool (BEAT): A Molecular Age Estimation Tool for Small Cetaceans. Frontiers in Marine Science. 82 2019; 6.

83 4. Würsig BG and Jefferson TA. (1990). Methods of photo-identification for small 84 cetaceans. Report of the International Whaling Commission, pp. 43-55

85 5. Wells RS. (2014). Social structure and life history of common bottlenose dolphins near

86 Sarasota Bay, Florida: Insights from four decades and five generations. In: Yamagiwa J,

87 Karczmarski, L., ed. Primates and Cetaceans: Field Research and Conservation of Complex

88 Mammalian Societies, Primatology Monographs: Springer), pp. 149-172.

89 6. Myrick AC. (1980). Examination of layered tissues of odontocetes for age determination

90 using polarized light microscopy. In: Perrin WF, Myrick Jr, A.C., ed. Age Determination of

91 Toothed Whales and Sirenians, pp. 105-112.

92 7. Hohn AA and Fernandez S. Biases in dolphin age structure due to age estimation 93 technique. Marine Mammal Science. 1999; 15(4):1124-1132.

94 8. Bada J, Brown S and Masters P. Age determination of marine mammals based on 95 aspartic acid racemization in the teeth and lens nucleus. Age Determination of Toothed Whales 96 and Sirenians Report of the International Whaling Commission, Special. 1980; (3):113-118.

$979 . \quad$ George JC, Bada J, Zeh J, Scott L, Brown SE, O'Hara T and Suydam R. Age and growth

98 estimates of bowhead whales (Balaena mysticetus) via aspartic acid racemization. Canadian 99 Journal of Zoology. 1999; 77(4):571-580.

100 10. Koopman HN, Iverson SJ and Gaskin DE. Stratification and age-related differences in 101 blubber fatty acids of the male harbour porpoise (Phocoena phocoena). J Comp Physiol B. 1996; 102 165(8):628-639.

103 11. Herman DP, Ylitalo GM, Robbins J, Straley JM, Gabriele CM, Clapham PJ, Boyer RH, 104 Tilbury KL, Pearce RW and Krahn MM. Age determination of humpback whales Megaptera 105 novaeangliae through blubber fatty acid compositions of biopsy samples. Marine Ecology 106 Progress Series. 2009; 392:277-293.

107 12. Marcoux M, Lesage V, Thiemann GW, Iverson SJ and Ferguson SH. Age estimation of 108 belugas, Delphinapterus leucas, using fatty acid composition: A promising method. Marine 109 Mammal Science. 2015; 31(3):944-962.

110 13. Stewart R, Campana S, Jones C and Stewart B. Bomb radiocarbon dating calibrates 111 beluga (Delphinapterus leucas) age estimates. Canadian Journal of Zoology. 2006; 84(12):18401121852. 
113 14. Olsen MT, Berube M, Robbins J and Palsboll PJ. Empirical evaluation of humpback whale 114 telomere length estimates; quality control and factors causing variability in the singleplex and 115 multiplex qPCR methods. BMC Genet. 2012; 13:77.

116 15. Herrman JM, Morey JS, Takeshita R, De Guise S, Wells RS, McFee W, Speakman T, 117 Townsend F, Smith CR, Rowles T and Schwacke L. Age determination of common bottlenose 118 dolphins (Tursiops truncatus) using dental radiography pulp:tooth area ratio measurements. 119 PLoS One. 2020; 15(11):e0242273.

120 16. Barratclough A, Sanz-Requena R, Marti-Bonmati L, Schmitt TL, Jensen E and Garcia-

121 Parraga D. Radiographic assessment of pectoral flipper bone maturation in bottlenose dolphins 122 (Tursiops truncatus), as a novel technique to accurately estimate chronological age. PLoS One. 123 2019; 14(9):e0222722.

124 17. Polanowski AM, Robbins J, Chandler D and Jarman SN. Epigenetic estimation of age in 125 humpback whales. Molecular ecology resources. 2014; 14(5):976-987.

126 18. Cole JJ, Robertson NA, Rather MI, Thomson JP, McBryan T, Sproul D, Wang T, Brock C, 127 Clark $\mathrm{W}$ and Ideker T. Diverse interventions that extend mouse lifespan suppress shared age128 associated epigenetic changes at critical gene regulatory regions. Genome biology. 2017;

129 18(1):1-16.

130 19. Wang T, Tsui B, Kreisberg JF, Robertson NA, Gross AM, Yu MK, Carter H, Brown-Borg $131 \mathrm{HM}$, Adams PD and Ideker T. Epigenetic aging signatures in mice livers are slowed by dwarfism, 132 calorie restriction and rapamycin treatment. Genome biology. 2017; 18(1):1-11.

133 20. Thompson MJ, Chwiałkowska K, Rubbi L, Lusis AJ, Davis RC, Srivastava A, Korstanje R, 134 Churchill GA, Horvath S and Pellegrini M. A multi-tissue full lifespan epigenetic clock for mice. 135 Aging (Albany NY). 2018; 10(10):2832.

136 21. Prado NA, Brown JL, Zoller JZ, Haghani A, Yao M, Bagryanova L, Campana M, Maldonado $137 \mathrm{JE}$, Raj K and Schmitt D. Epigenetic clock and methylation studies in elephants. bioRxiv. 2020.

138 22. Lu AT, Fei Z, Haghani A, Robeck TR, Zoller JA, Li CZ, Zhang J, Ablaeva J, Adams DM and 139 Almunia J. Universal DNA methylation age across mammalian tissues. bioRxiv. 2021.

140 23. Bors EK, Baker CS, Wade PR, O'Neill KB, Shelden KE, Thompson MJ, Fei Z, Jarman S and 141 Horvath $S$. An epigenetic clock to estimate the age of living beluga whales. Evolutionary 142 Applications. 2020.

143 24. Smith ZD and Meissner A. DNA methylation: roles in mammalian development. Nature 144 Reviews Genetics. 2013; 14(3):204-220.

145 25. Rakyan VK, Down TA, Maslau S, Andrew T, Yang T-P, Beyan H, Whittaker P, McCann OT, 146 Finer $S$ and Valdes AM. Human aging-associated DNA hypermethylation occurs preferentially at 147 bivalent chromatin domains. Genome research. 2010; 20(4):434-439.

148 26. Teschendorff AE, Menon U, Gentry-Maharaj A, Ramus SJ, Weisenberger DJ, Shen $H$, 149 Campan M, Noushmehr H, Bell CG and Maxwell AP. Age-dependent DNA methylation of genes 150 that are suppressed in stem cells is a hallmark of cancer. Genome research. 2010; 20(4):440151446.

152 27. Horvath S and Raj K. DNA methylation-based biomarkers and the epigenetic clock theory 153 of ageing. Nature Reviews Genetics. 2018; 19(6):371-384. 
154 28. Bell CG, Lowe R, Adams PD, Baccarelli AA, Beck S, Bell JT, Christensen BC, Gladyshev VN, 155 Heijmans BT and Horvath S. DNA methylation aging clocks: challenges and recommendations. 156 Genome biology. 2019; 20(1):1-24.

157 29. Zhang Q, Vallerga CL, Walker RM, Lin T, Henders AK, Montgomery GW, He J, Fan D, 158 Fowdar J, Kennedy M, Pitcher T, Pearson J, Halliday G, et al. Improved precision of epigenetic 159 clock estimates across tissues and its implication for biological ageing. Genome Med. 2019; 160 11(1):54.

161 30. Field AE, Robertson NA, Wang T, Havas A, Ideker T and Adams PD. DNA Methylation 162 Clocks in Aging: Categories, Causes, and Consequences. Mol Cell. 2018; 71(6):882-895.

163 31. Trego ML, Kellar NM and Danil K. Validation of blubber progesterone concentrations for 164 pregnancy determination in three dolphin species and a porpoise. PLoS One. 2013; 8(7):e69709. 32. Galligan TM, Balmer BC, Schwacke LH, Bolton JL, Quigley BM, Rosel PE, Ylitalo GM and Boggs AS. Examining the relationships between blubber steroid hormones and persistent organic pollutants in common bottlenose dolphins. Environmental Pollution. 2019; 249:982991.

33. Parsons KM. (2002). The use of molecular and observational data to infer the structuring of bottlenose dolphin populations. University of Aberdeen).

171 34. Chen BH, Marioni RE, Colicino E, Peters MJ, Ward-Caviness CK, Tsai P-C, Roetker NS, Just 172 AC, Demerath EW and Guan W. DNA methylation-based measures of biological age: meta173 analysis predicting time to death. Aging (Albany NY). 2016; 8(9):1844.

174 35. Perna L, Zhang Y, Mons U, Holleczek B, Saum K-U and Brenner H. Epigenetic age 175 acceleration predicts cancer, cardiovascular, and all-cause mortality in a German case cohort.

176 Clinical epigenetics. 2016; 8(1):1-7.

177 36. Marioni RE, Shah S, McRae AF, Ritchie SJ, Muniz-Terrera G, Harris SE, Gibson J, Redmond 178 P, Cox SR and Pattie A. The epigenetic clock is correlated with physical and cognitive fitness in 179 the Lothian Birth Cohort 1936. International journal of epidemiology. 2015; 44(4):1388-1396.

180 37. Lu AT, Quach A, Wilson JG, Reiner AP, Aviv A, Raj K, Hou L, Baccarelli AA, Li Y and 181 Stewart JD. DNA methylation GrimAge strongly predicts lifespan and healthspan. Aging (Albany 182 NY). 2019; 11(2):303.

183 38. Rieger E, Bijl JJ, van Oostveen JW, Soyer HP, Oudejans CB, Jiwa NM, Walboomers JM and

184 Meijer CJ. Expression of the homeobox gene HOXC4 in keratinocytes of normal skin and 185 epithelial skin tumors is correlated with differentiation. Journal of investigative dermatology. 186 1994; 103(3):341-346.

187 39. Adelman ER, Huang H-T, Roisman A, Olsson A, Colaprico A, Qin T, Lindsley RC, Bejar R, 188 Salomonis N and Grimes HL. Aging human hematopoietic stem cells manifest profound 189 epigenetic reprogramming of enhancers that may predispose to leukemia. Cancer discovery.

190 2019; 9(8):1080-1101.

191 40. Vidaki A, Ballard D, Aliferi A, Miller TH, Barron LP and Syndercombe Court D. DNA 192 methylation-based forensic age prediction using artificial neural networks and next generation 193 sequencing. Forensic Sci Int Genet. 2017; 28:225-236. 
194 41. Maat GJ, Maes A, Aarents MJ and Nagelkerke NJ. Histological Age Prediction from the 195 Femur in a Contemporary Dutch Sample* The decrease of nonremodeled bone in the anterior 196 cortex. Journal of forensic sciences. 2006; 51(2):230-237.

197 42. Huang Y, Yan J, Hou J, Fu X, Li L and Hou Y. Developing a DNA methylation assay for human age prediction in blood and bloodstain. Forensic Science International: Genetics. 2015; $17: 129-136$

200 43. Gronniger E, Weber B, Heil O, Peters N, Stab F, Wenck H, Korn B, Winnefeld M and Lyko 201 F. Aging and chronic sun exposure cause distinct epigenetic changes in human skin. PLoS Genet. 202 2010; 6(5):e1000971.

203 44. Petit MM, Schoenmakers EF, Huysmans C, Geurts JM, Mandahl N and Van de Ven WJ. 204 LHFP, a Novel Translocation Partner Gene ofHMGICin a Lipoma, Is a Member of a New Family ofLHFP-like Genes. Genomics. 1999; 57(3):438-441. 45. Wu M, Tian H-L, Liu X, Lai JHC, Du S and Xia J. Impairment of inhibitory synapse formation and motor behavior in mice lacking the NL2 binding partner LHFPL4/GARLH4. Cell reports. 2018; 23(6):1691-1705.

209 46. Taracha A, Kotarba G and Wilanowski T. Neglected functions of TFCP2/TFCP2L1/UBP1 transcription factors may offer valuable insights into their mechanisms of action. International journal of molecular sciences. 2018; 19(10):2852.

212 47. Kotarba G, Krzywinska E, Grabowska Al, Taracha A and Wilanowski T.

213 TFCP2/TFCP2L1/UBP1 transcription factors in cancer. Cancer letters. 2018; 420:72-79. Suz12 is required for embryonic stem cell differentiation. Molecular and cellular biology. 2007; 27(10):3769-3779.

217 49. Takahiro Ito YVT, Shane A. Evans, Nicola Neretti, John M. Sedivy,. Regulation of Cellular 218 Senescence by Polycomb Chromatin Modifiers through Distinct DNA Damage- and Histone Methylation-Dependent Pathways. Cell Reports. 2018; 22(13):3480-3492.

220 50. Kao Y-C, Sung Y-S, Zhang L, Jungbluth AA, Huang S-C, Argani P, Agaram NP, Zin A, Alaggio $221 \mathrm{R}$ and Antonescu CR. BCOR overexpression is a highly sensitive marker in round cell sarcomas

222 with BCOR genetic abnormalities. The American journal of surgical pathology. 2016;

223 40(12):1670.

224 51. Guo C, Eckler MJ, McKenna WL, McKinsey GL, Rubenstein JL and Chen B. Fezf2

225 expression identifies a multipotent progenitor for neocortical projection neurons, astrocytes, 226 and oligodendrocytes. Neuron. 2013; 80(5):1167-1174.

227 52. Pisano C, Merlini L, Penco S, Cincinelli R, Darwiche N, Guglielmi MB, La Porta I, Signorino 228 G, De Rubis G and Colelli F. (2018). Preclinical antitumor activity of novel DNA polymerase 1 229 (POLA1) inhibitors. Cancer Research: AMER ASSOC CANCER RESEARCH 615 CHESTNUT ST, 17TH 230 FLOOR, PHILADELPHIA, PA ...).

231 53. Li H, Zhu Y, Morozov YM, Chen X, Page SC, Rannals MD, Maher BJ and Rakic P.

232 Disruption of TCF4 regulatory networks leads to abnormal cortical development and mental 233 disabilities. Molecular psychiatry. 2019; 24(8):1235-1246.

234 54. de Vicente JC, Donate-Pérez del Molino P, Rodrigo JP, Allonca E, Hermida-Prado F, 235 Granda-Díaz R, Rodríguez Santamarta T and García-Pedrero JM. SOX2 expression is an 
independent predictor of oral cancer progression. Journal of clinical medicine. 2019;

8(10):1744.

55. Nthiga TM. Role of CALCOCO1 in scaling down endoplasmic reticulum and Golgi by autophagy. 2020.

240 56. Prado NA, Brown JL, Zoller JA, Haghani A, Yao M, Bagryanova LR, Campana MG,

241 Maldonado JE, Raj K, Schmitt D, Robeck TR and Horvath S. 2020.

242 57. Arneson A, Haghani A, Thompson MJ, Pellegrini M, Kwon SB, Vu HT, Li CZ, Lu AT, Barnes

243 B and Hansen KD. A mammalian methylation array for profiling methylation levels at conserved

244 sequences. bioRxiv. 2021:2021.2001. 2007.425637.

245 58. Zhou W, Triche TJ, Jr., Laird PW and Shen H. SeSAMe: reducing artifactual detection of

246 DNA methylation by Infinium BeadChips in genomic deletions. Nucleic Acids Res. 2018;

247 46(20):e123.

248 59. Friedman J, Hastie T and Tibshirani R. Regularization paths for generalized linear models via coordinate descent. Journal of statistical software. 2010; 33(1):1.

$25060 . \quad$ Shao J. Linear model selection by cross-validation. Journal of the American statistical 251 Association. 1993; 88(422):486-494.

252 61. Zhang P. Model selection via multifold cross validation. The annals of statistics.

253 1993:299-313.

254 62. Zou H and Hastie T. Regularization and variable selection via the elastic net. Journal of 255 the Royal Statistical Society: Series B (Statistical Methodology). 2005; 67(2):301-320.

256 63. Halvardson J, Zhao JJ, Zaghlool A, Wentzel C, Georgii-Hemming P, Månsson E, Sävmarker

$257 \mathrm{HE}$, Brandberg G, Zander CS and Thuresson A-C. Mutations in HECW2 are associated with

258 intellectual disability and epilepsy. Journal of medical genetics. 2016; 53(10):697-704.

259 64. Krishnamoorthy V, Khanna R and Parnaik VK. E3 ubiquitin ligase HECW2 targets PCNA

260 and lamin B1. Biochimica et Biophysica Acta (BBA)-Molecular Cell Research. 2018;

261 1865(8):1088-1104.

262 65. Luoma LM and Berry FB. Molecular analysis of NPAS3 functional domains and variants.

263 BMC molecular biology. 2018; 19(1):1-19.

264 66. Llano-Diez M, Fury W, Okamoto H, Bai Y, Gromada J and Larsson L. RNA-sequencing reveals altered skeletal muscle contraction, E3 ligases, autophagy, apoptosis, and chaperone expression in patients with critical illness myopathy. Skeletal Muscle. 2019; 9(1):9.

67. Lefebvre V, Li P and de Crombrugghe B. A new long form of Sox5 (L-Sox5), Sox6 and Sox9 are coexpressed in chondrogenesis and cooperatively activate the type II collagen gene. The EMBO Journal. 1998; 17(19):5718-5733. Abbadie C, Carling D and De Launoit Y. Regulation of ploidy and senescence by the AMPKrelated kinase NUAK1. The EMBO journal. 2010; 29(2):376-386. 
276 70. Angulo JC, Andrés G, Ashour N, Sánchez-Chapado M, López JI and Ropero S.

277 Development of castration resistant prostate cancer can be predicted by a DNA

278 hypermethylation profile. The Journal of urology. 2016; 195(3):619-626.

279 71. Cai C, Hsieh C-L, Omwancha J, Zheng Z, Chen S-Y, Baert J-L and Shemshedini L. ETV1 is a 280 novel androgen receptor-regulated gene that mediates prostate cancer cell invasion. Molecular 281 endocrinology. 2007; 21(8):1835-1846.

282 72. Zeng Z, Xie D and Gong J. Genome-wide identification of CpG island methylator 283 phenotype related gene signature as a novel prognostic biomarker of gastric cancer. PeerJ.

284 2020; 8:e9624.

285 73. Herault Y, Hraba-Renevey S, Van der Hoeven F and Duboule D. Function of the Evx-2 286 gene in the morphogenesis of vertebrate limbs. The EMBO Journal. 1996; 15(23):6727-6738.

287 74. Sheikholeslami S, Azizi F, Ghasemi A, Alibakhshi A, Parsa H, Tavangar SM, Shivaee S, Zarif 288 Yeganeh M, Hedayati M and Teimoori-Toolabi L. NOL4 is Downregulated and Hyper-Methylated 289 in Papillary Thyroid Carcinoma Suggesting Its Role as a Tumor Suppressor Gene. International 290 Journal of Endocrinology and Metabolism. 2020; 18(4).

291 75. Demokan S, Chuang AY, Pattani KM, Sidransky D, Koch W and Califano JA. Validation of 292 nucleolar protein 4 as a novel methylated tumor suppressor gene in head and neck cancer.

293 Oncology reports. 2014; 31(2):1014-1020.

294 76. Pangas SA, Choi Y, Ballow DJ, Zhao Y, Westphal H, Matzuk MM and Rajkovic A.

295 Oogenesis requires germ cell-specific transcriptional regulators Sohlh1 and Lhx8. Proceedings of 296 the National Academy of Sciences. 2006; 103(21):8090-8095.

297 77. Zhao Y, Marín O, Hermesz E, Powell A, Flames N, Palkovits M, Rubenstein JL and 298 Westphal H. The LIM-homeobox gene Lhx8 is required for the development of many cholinergic 299 neurons in the mouse forebrain. Proceedings of the National Academy of Sciences. 2003;

300 100(15):9005-9010.

301 